\title{
Photon capture cones and embedding diagrams of the Ernst spacetimet
}

\author{
Z Stuchlík@ and S Hledík \\ Institute of Physics, Faculty of Philosophy and Science, Silesian University \\ in Opava, Bezručovo nám. 13, CZ-746 01 Opava, Czech Republic
}

\begin{abstract}
The differences between the character of the Schwarzschild and Ernst spacetimes are illustrated by comparing the photon capture cones, and the embedding diagrams of the $t=$ const sections of the equatorial planes of both the ordinary and optical reference geometry of these spacetimes. The non-flat asymptotic character of the Ernst spacetime reflects itself in two manifest facts: the escape photon cones correspond to purely outward radial direction, and the embedding diagrams of both the ordinary and optical geometry shrink to zero radius asymptotically. Using the properties of the embedding diagrams, regions of these spacetimes which could have similar character are estimated, and it is argued that they can exist for the Ernst spacetimes with a sufficiently low strength of the magnetic field.
\end{abstract}

PACS numbers: 98.80.-k, 95.30.Sf, 04.70.-s, 04.20.Jb

$\ddagger$ Published in Class. Quantum Grav. 16 (1999), pp. 1377-1387.

$\S$ E-mail address: Zdenek.Stuchlik@fpf.slu.cz

|| E-mail address: Stanislav.Hledik@fpf.slu.cz 


\section{Introduction}

The static Ernst spacetime [1] is the only exact solution of Einstein's equations known to represent the spacetime of a spherically symmetric massive body or black hole of mass $M$ immersed in an otherwise homogeneous magnetic field. If the magnetic field disappears, the geometry simplifies to the Schwarzschild geometry. Therefore, sometimes the Ernst spacetime is called magnetized Schwarzschild spacetime. Usually it is believed that for extended structures like galaxies both the effects of general relativity and the role of a magnetic field can be ignored. However, in the case of active galactic nuclei with a huge central black hole and an important magnetic field, the Ernst spacetime can represent some relevant properties of the galactic structures. Therefore, it could even be astrophysically important to discuss and illustrate the properties of the Ernst spacetime.

The Ernst spacetime has the important property that it is not asymptotically flat. Far from the black hole, the spacetime is closely related to Melvin's magnetic universe, representing a cylindrically symmetric spacetime filled with an uniform magnetic field only. The Ernst spacetime is axially symmetric, and its structure corresponds to the structure of the Schwarzschild spacetime only along its axis of symmetry. Off the axis, the differences are very spectacular, and we shall demonstrate them for the equatorial plane, which is the symmetry plane of the spacetime.

Motion of test particles and photons in the Ernst spacetime was discussed by number of authors [2, 3, 4, 5, 6], and number of properties different from those of the Schwarzschild spacetime were discovered. For example, if the magnetic field is weak enough, a stable circular photon orbit can exist behind the unstable circular photon orbit located nearby the black-hole horizon. We illustrate the impact of this fact on the structure of photon capture cones, and we compare them with the corresponding ones in the Schwarzschild spacetime in Section [2 Further we illustrate properties of the Ernst spacetime by comparing the embedding diagrams of its $t=$ const sections with embedding diagrams of the Schwarzschild spacetime both for ordinary geometry and optical reference geometry in Section 3 . These embedding diagrams give in an illustrative way information on changes of the spacetime structure caused by the presence of an uniform magnetic field about the central black hole. The most important change comes from the asymptotic behaviour of the spacetime which is manifested by the fact that the radius of the embedding diagrams shrink asymptotically to zero. Finally, in Section 4, we estimate which regions of the Ernst and Schwarzschild spacetimes could be considered as having similar character.

\section{Photon capture cones}

In the standard Schwarzschild coordinates $(t, r, \theta, \phi)$, and the geometric system of units $(c=G=1)$, the Ernst spacetime is determined by the line element

$\mathrm{d} s^{2}=\Lambda^{2}\left[-\left(1-\frac{2 M}{r}\right) \mathrm{d} t^{2}+\left(1-\frac{2 M}{r}\right)^{-1} \mathrm{~d} r^{2}+r^{2} \mathrm{~d} \theta^{2}\right]+\frac{r^{2} \sin ^{2} \theta}{\Lambda^{2}} \mathrm{~d} \phi^{2}$

where

$$
\Lambda \equiv 1+B^{2} r^{2} \sin ^{2} \theta
$$

with $M$ (mass) and $B$ (strength of the magnetic field) being the parameters of the spacetime. The dimensionless combination of these parameters, $B M$, has to be very 
small in astrophysically realistic situations. Using, for a moment, the c.g.s. units, we find

$$
B M \sim 2 \times 10^{-53} B_{\mathrm{cgs}} M_{\mathrm{cgs}}
$$

where $M=M_{\mathrm{cgs}} G / c^{2}$, and $B=B_{\mathrm{cgs}} G^{1 / 2} / c^{2}$. Then for a black hole with $M=10^{9} M_{\odot}$, typical for active galactic nuclei, the parameter $B M \sim 1$ could be obtained for an extremely strong magnetic field of $B_{\mathrm{cgs}} \sim 10^{11}$ Gauss.

In the following discussion we put $M=1$ for simplicity. Then the coordinates $r, t$ become dimensionless, and $B M \rightarrow B$.

In the simple case of the motion of photons in the equatorial plane $(\theta=\pi / 2)$, the geodesic equation $\mathrm{D} p^{\mu} / \mathrm{d} \lambda=0$ with the normalization condition $p^{\mu} p_{\mu}=0$ can be solved easily. (The 4-momentum is $p^{\mu}=\mathrm{d} x^{\mu} / \mathrm{d} \lambda$, and $\lambda$ is the affine parameter along the geodesic.) Except the constant latitude $\theta=\pi / 2$, there are constants of the motion connected to the time and azimuthal Killing vectors, $\partial / \partial t$ and $\partial / \partial \phi$, of the spacetime:

$$
-\mathcal{E}=p_{t}=g_{t \mu} p^{\mu}
$$

and

$$
\Phi=p_{\phi}=g_{\phi \mu} p^{\mu} .
$$

Using the normalization condition, the equations of the photon motion can be given in the following form

$$
\begin{aligned}
& \left(p^{r}\right)^{2}=\left(\frac{\mathrm{d} r}{\mathrm{~d} \lambda}\right)^{2}=\frac{\mathcal{E}^{2}}{\Lambda^{4}}-\frac{1-2 / r}{r^{2}} \Phi^{2} \\
& p^{\theta}=\frac{\mathrm{d} \theta}{\mathrm{d} \lambda}=0 \\
& p^{\phi}=\frac{\mathrm{d} \phi}{\mathrm{d} \lambda}=\frac{\Lambda^{2}}{r^{2}} \Phi \\
& p^{t}=\frac{\mathrm{d} t}{\mathrm{~d} \lambda}=\frac{\mathcal{E}}{\Lambda^{2}(1-2 / r)} .
\end{aligned}
$$

Introducing an impact parameter by the relation

$$
l=\frac{\Phi}{\mathcal{E}}
$$

and making a rescaling of the affine parameter by $\mathcal{E} \lambda \rightarrow \lambda$, we find from the radial equation (6) that the photon motion is allowed in regions where

$$
l^{2} \leq l_{\mathrm{R}}^{2}(r ; B) \equiv \frac{r^{2}}{\Lambda^{4}(1-2 / r)} .
$$

The turning points of the radial motion of a photon with impact parameter $l$ are given by $l^{2}=l_{\mathrm{R}}^{2}(r ; B)$.

As in the Schwarzschild spacetime, the function $l_{\mathrm{R}}^{2}(r ; B)$ diverges at $r=2$, giving location of the event horizon of the Ernst spacetime. However, it behaves in a totally different manner asymptotically: $l_{\mathrm{R}}^{2} \rightarrow 0$ for $r \rightarrow \infty$.

Since

$$
\frac{\partial l_{\mathrm{R}}^{2}}{\partial r}=\frac{2 r^{2}\left(r-3+5 r^{2} B^{2}-3 r^{3} B^{2}\right)}{\left(1+B^{2} r^{2}\right)^{5}(r-2)^{2}}
$$




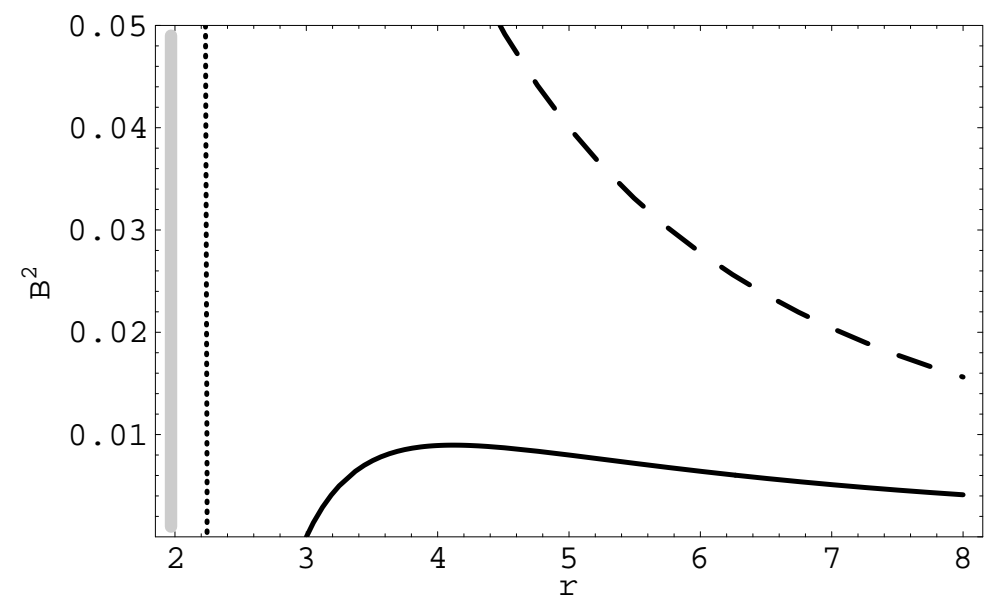

Figure 1. The behaviour of $B_{\mathrm{ph}}^{2}$ (solid curve), extreme of the ordinary embeddings $B_{\max }^{2}$ (dashed curve), defined by Eq. 33 and the radii of the limit of the embeddability of the optical geometry, determined by solving Eq. (50) numerically (dotted curve) as functions of $r$.

the photon circular orbits, located at the local extrema of $l_{\mathrm{R}}^{2}$, are determined by the condition

$$
3 B^{2} r^{3}-5 B^{2} r^{2}-r+3=0
$$

or, equivalently, by the relation

$$
B^{2}=B_{\mathrm{ph}}^{2}(r) \equiv \frac{r-3}{r^{2}(3 r-5)} .
$$

The function $B_{\mathrm{ph}}^{2}(r)$ is given in Fig. 1. It is defined at $r \geq 3$; as expected, for $B=0$ we obtain the circular photon orbit of the Schwarzschild spacetime. Its maximum is located at $r_{\mathrm{c}}=(8+\sqrt{19}) / 3$, and it defines a critical value of the magnetic-field parameter:

or

$$
B_{\mathrm{c}}^{2}=\frac{3(\sqrt{19}-1)}{(8+\sqrt{19})^{2}(3+\sqrt{19})} \approx 0.008965
$$

$$
B_{\mathrm{c}} \approx 0.0947 .
$$

If $B<B_{\mathrm{c}}$, there exist two circular photon orbits. The inner one at $r_{\mathrm{i}}$ is unstable with respect to radial perturbations, the outer one at $r_{\mathrm{o}}$ is stable. If $B=B_{\mathrm{c}}$, these two circular orbits coalesce. If $B>B_{\mathrm{c}}$, no photon circular orbits can exist (see Fig. 2). For realistic magnetic fields, $B \ll 1$, we find

$$
r_{\mathrm{i}} \sim 3, r_{\mathrm{o}} \sim \frac{2}{\sqrt{6} B} .
$$

We can see that if $B<B_{\mathrm{c}}$, bound photons orbits exist around the outer, stable, photon circular orbit, which are not captured by the hole, and cannot escape to infinity. Such orbits can extend down to the unstable photon circular orbit. On the other hand, purely radially directed photons with a zero impact parameter can only escape to infinity, if they are outward directed initially. If $B>B_{\mathrm{c}}$, all photons are captured by the hole, except the outward directed radial photons, which escape to infinity. 


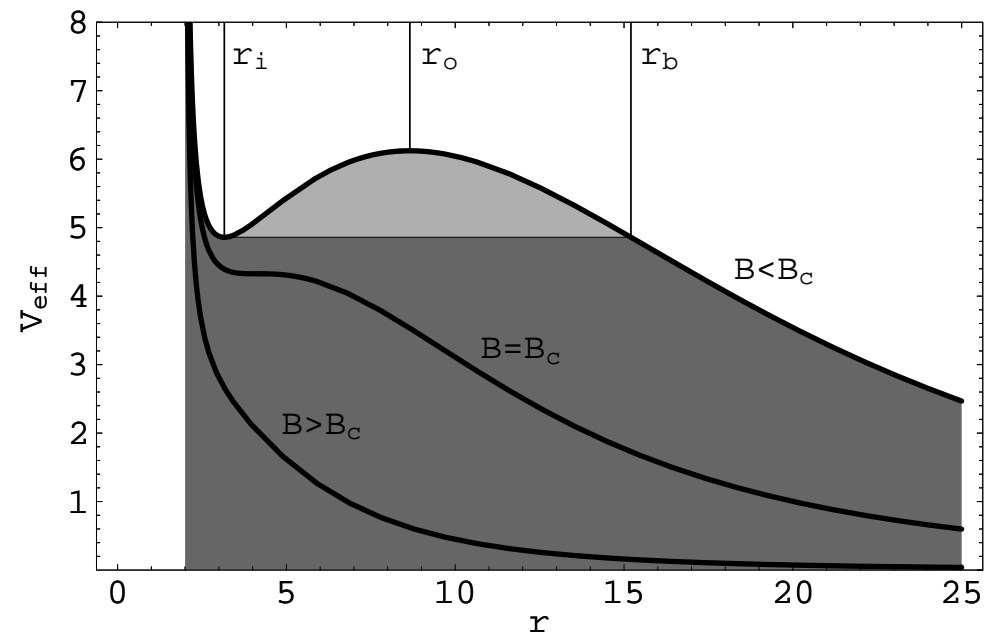

Figure 2. The function $l_{\mathrm{R}}^{2}(r ; B)$ governing the photon motion is drawn for $B=0.2>B_{\mathrm{c}}, B=B_{\mathrm{c}} \approx 0.0947$ and $B=0.06<B_{\mathrm{c}}$. Photons having its radius of emission $r$ and the impact parameter $l$ in the dark grey area are captured, the light grey area represents bound photons.

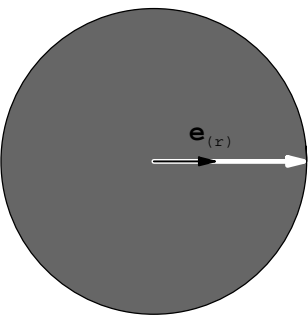

$r \leq r_{\mathrm{i}} \approx 3.161$

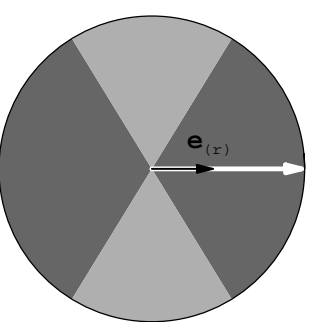

$r=12$

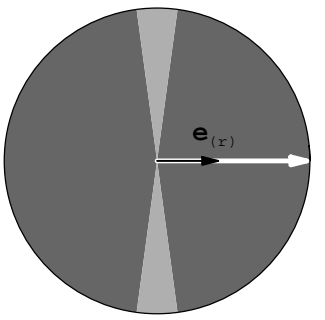

$r=3.5$

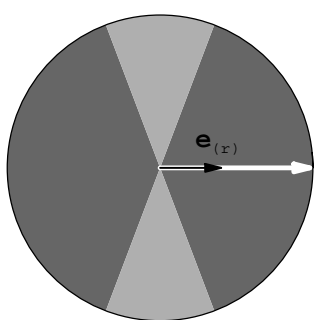

$r=14$

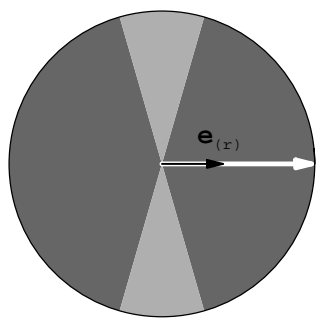

$r=4$

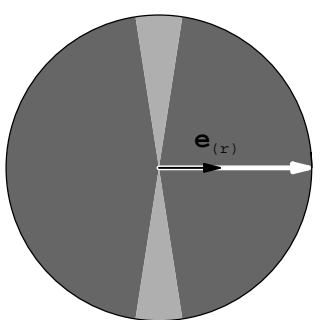

$r=15$

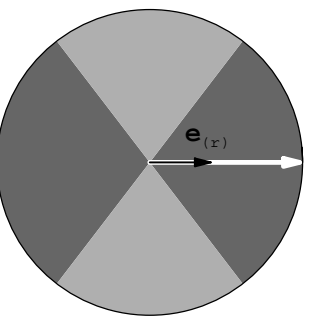

$r=r_{\mathrm{o}} \approx 8.656$

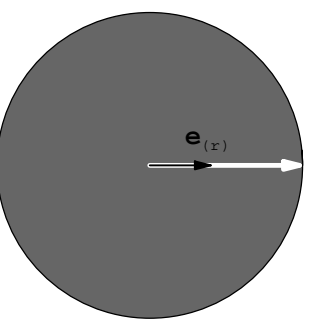

$r \geq r_{\mathrm{b}} \approx 15.205$

Figure 3. Directions of captured (dark grey) and bound (light grey) photons for several values of the radius of a static observer for $B=0.06<B_{\mathrm{c}}$. Note that if $2<r \leq r_{\mathrm{i}} \approx 3.161$ or $r \geq r_{\mathrm{b}} \approx 15.205$, all photons are captured. Only the purely radially outward emitted photons (white arrow) can escape to infinity. Escape angles for the Schwarzschild black hole can be found, e.g., in 7 .

Now, we can determine the photon capture cones of static observers. In the Ernst spacetime, we introduce the tetrad of differential forms $\omega_{\mu}^{(\alpha)}$ related to the 
static observers by the relations

$$
\begin{aligned}
& \omega^{(t)}=\left(1+B^{2} r^{2}\right) \sqrt{1-\frac{2}{r}} \mathrm{~d} t \\
& \omega^{(r)}=\left(1+B^{2} r^{2}\right)\left(1-\frac{2}{r}\right)^{-1 / 2} \mathrm{~d} r \\
& \omega^{(\theta)}=\left(1+B^{2} r^{2}\right) r \mathrm{~d} \theta \\
& \omega^{(\phi)}=\frac{r}{1+B^{2} r^{2}} \mathrm{~d} \phi .
\end{aligned}
$$

The tetrad of vectors $e_{(\alpha)}^{\mu}$, dual to the tetrad of the differential forms, is related in the standard manner and will not be explicitly given here. The components of photon's 4-momentum, as measured by the static observers, are determined by the relations

$$
\begin{aligned}
& p_{(\alpha)}=p_{\mu} e_{(\alpha)}^{\mu} \\
& p^{(\alpha)}=p^{\mu} \omega_{\mu}^{(\alpha)} .
\end{aligned}
$$

and its directional angle $\psi$, related to the outward radial direction, is given by the relation

$$
\sin \psi=\frac{p^{(\phi)}}{p^{(t)}} .
$$

Since the relevant components of the 4-momentum are

$$
\begin{aligned}
& p^{(t)}=\frac{\mathcal{E}}{\left(1+B^{2} r^{2}\right) \sqrt{1-2 / r}} \\
& p^{(\phi)}=\frac{\left(1+B^{2} r^{2}\right)}{r} \Phi
\end{aligned}
$$

we arrive at the final formula

$$
\sin \psi=\left(1+B^{2} r^{2}\right) \sqrt{1-\frac{2}{r}} \frac{l}{r} .
$$

The photon capture cones can be determined by using the function $l_{\mathrm{R}}^{2}(r ; B)$ governing the photon motion. In establishing the directional angle of marginally captured photons $\psi_{\mathrm{c}}$ for $B<B_{\mathrm{c}}$, we have to use impact parameter $l_{\mathrm{c}}$ of the unstable circular photon orbit, which plays a crucial role. Directions of captured and bound photons are determined by a numerical procedure, and they are depicted in a sequence of figures for typical values of the radius of static observers in the field of a black hole with the parameter $B=0.06<B_{\mathrm{c}}$ (see Fig. 3). Notice that for impact parameters satisfying the condition $0<l^{2}<l_{\mathrm{c}}^{2}$, all the photons are captured by the hole. The bound orbits can exist between the radii $r_{\mathrm{i}}$ and $r_{\mathrm{b}}$; the definition of the radius $r_{\mathrm{b}}$ is clear from Fig. 2. At radii $r_{\mathrm{i}}<r<r_{\mathrm{b}}$, regions corresponding to bound orbits appear around directions orthogonal to the outward radial direction. The regions are most extended at $r=r_{\mathrm{o}}$, and they shrink to zero for $r \rightarrow r_{\mathrm{i}}$ and $r \rightarrow r_{\mathrm{b}}$.

If $B>B_{\mathrm{c}}$, the situation is quite simple. At each radii there are only captured photons, except the purely radially outward directed photons, which reach infinity asymptotically. 


\section{Embedding diagrams}

It is well known that embedding diagrams of some representative sections of blackhole spacetimes give an useful intuitive understanding of the basic properties of these spacetimes (see, e.g., [7, 8, 9, 10]). Because we are familiar to the Euclidean space, usually two-dimensional sections of the black-hole spacetimes are embedded into the three-dimensional space with the line element expressed in the cylindrical coordinates:

$$
\mathrm{d} s^{2}=\mathrm{d} \rho^{2}+\rho^{2} \mathrm{~d} \phi^{2}+\mathrm{d} z^{2} .
$$

Of course, embeddings into some other spaces can also give interesting information. However, here we restrict our attention to the most straightforward Euclidean case.

The time Killing vector field $\partial / \partial t$ of the Ernst spacetime leads to a privileged space-like sections - namely the hypersurfaces $t=$ const. We shall do the embedding for the equatorial plane of these hypersurfaces. Then the line element reads

$$
\mathrm{d} l^{2}=\frac{\left(1+B^{2} r^{2}\right)^{2}}{1-2 / r} \mathrm{~d} r^{2}+\frac{r^{2}}{\left(1+B^{2} r^{2}\right)^{2}} \mathrm{~d} \phi^{2} .
$$

We have to find a surface $z=z(\rho)$ in the three dimensional Euclidean space, which is isometric to the two-dimensional space given by (29). Therefore, we identify the space with the line element

$$
\mathrm{d} l_{(\mathrm{E})}^{2}=\left[1+\left(\frac{\mathrm{d} z}{\mathrm{~d} \rho}\right)^{2}\right] \mathrm{d} \rho^{2}+\rho^{2} \mathrm{~d} \phi^{2} .
$$

Clearly, the azimuthal coordinates can be identified. But, contrary to the Schwarzschild case, the radial coordinates cannot be identified directly. There is

$$
\rho=\frac{r}{1+B^{2} r^{2}}
$$

and due to this relation the Ernst and Schwarzschild embeddings are totally different in the asymptotic region of $r \rightarrow \infty$. Really, $\rho \rightarrow 0$ for $r \rightarrow \infty$, and the Ernst embedding diagram shrinks to zero radius asymptotically. Because there is

$$
\frac{\mathrm{d} \rho}{\mathrm{d} r}=\frac{1-B^{2} r^{2}}{\left(1+B^{2} r^{2}\right)^{2}}
$$

the maximum radius of the embedding diagram corresponds to an Ernst radial coordinate

$$
r_{\max }(B)=\frac{1}{B}
$$

and an Euclidean radial coordinate

$$
\rho_{\max }(B)=\frac{1}{2 B} \text {. }
$$

The embedding formula $z=z(\rho)$ is given by the relation

$$
\left(\frac{\mathrm{d} z}{\mathrm{~d} \rho}\right)^{2}=\frac{\left(1+B^{2} r^{2}\right)^{2}}{1-2 / r}\left(\frac{\mathrm{d} r}{\mathrm{~d} \rho}\right)^{2}-1 .
$$

However, it is much simpler to express the embedding formula in a parametric form, using $r$ as a parameter. From (32) and (35) we arrive at

$$
\left(\frac{\mathrm{d} z}{\mathrm{~d} r}\right)^{2}=\frac{\left(1+B^{2} r^{2}\right)^{6}-\left(1-B^{2} r^{2}\right)^{2}(1-2 / r)}{(1-2 / r)\left(1+B^{2} r^{2}\right)^{4}} .
$$


The embedding (or reality) condition $(\mathrm{d} z / \mathrm{d} r)^{2} \geq 0$ implies two relations to be satisfied:

$$
\begin{aligned}
& \left(1+B^{2} r^{2}\right)^{6}-\left(1-B^{2} r^{2}\right)^{2}\left(1-\frac{2}{r}\right) \geq 0 \\
& r-2 \geq 0 .
\end{aligned}
$$

A numerical analysis shows that the first condition is satisfied at $r \geq 2$ for arbitrary $B^{2}>0$. The embedding diagrams has the same character for all values of the parameter $B$. We present them in Fig. 4. For comparison with the Schwarzschild case, we give the functions $z(\rho)$ in Fig. 5 .

Many relevant properties of black-hole spacetimes are related to the optical reference geometry [11, 12, 13, 14, 15, 16. The optical geometry can be introduced by the $(3+1)$ conformal splitting

$$
\mathrm{d} s^{2}=\mathrm{e}^{2 \Psi}\left(-\mathrm{d} t^{2}+\mathrm{d} \tilde{l}^{2}\right)
$$

where

$$
\mathrm{d} \tilde{l}^{2}=\tilde{g}_{i k} \mathrm{~d} x^{i} \mathrm{~d} x^{k}
$$

and $i, k=1,2,3$. In the case of the Ernst metric we find

$$
\begin{aligned}
& \mathrm{e}^{2 \Psi}=\Lambda^{2}\left(1-\frac{2}{r}\right) \\
& \tilde{g}_{r r}=\left(1-\frac{2}{r}\right)^{-2} \\
& \tilde{g}_{\theta \theta}=r^{2}\left(1-\frac{2}{r}\right) \\
& \tilde{g}_{\phi \phi}=\frac{r^{2} \sin ^{2} \theta}{\Lambda^{4}(1-2 / r)} .
\end{aligned}
$$

In the equatorial plane the line element is given by the relation

$$
\mathrm{d} \tilde{l}^{2}=\frac{\mathrm{d} r^{2}}{(1-2 / r)^{2}}+\frac{r^{2}}{\left(1+B^{2} r^{2}\right)^{4}(1-2 / r)} \mathrm{d} \phi^{2} .
$$

This have to be identified with the line element (30). We identify the azimuthal coordinates again, and for the radial coordinates we find the formulae

$$
\rho=\frac{r}{\left(1+B^{2} r^{2}\right)^{2} \sqrt{1-2 / r}}
$$

and

$$
\frac{\mathrm{d} \rho}{\mathrm{d} r}=\frac{1-3 / r+B^{2} r(5-3 r)}{\left(1+B^{2} r^{2}\right)^{3}(1-2 / r)^{3 / 2}} .
$$

We can see that also for the optical geometry of the Ernst spacetime, the embedding diagrams shrink to zero radius asymptotically. Further, there is $\mathrm{d} \rho / \mathrm{d} r=0$ just at the loci of the circular photon orbits. Of course, it is simply given by the fact that

$$
\tilde{g}_{\phi \phi}=l_{\mathrm{R}}^{2}
$$

at the equatorial plane. (Note that this is a general property of spherically symmetric spacetimes [10.) The embedding formula can be effectively treated in the parametric form:

$$
\left(\frac{\mathrm{d} z}{\mathrm{~d} r}\right)^{2}=\frac{(1-2 / r)\left(1+B^{2} r^{2}\right)^{6}-\left[1-3 / r+B^{2} r(5-3 r)\right]^{2}}{(1-2 / r)^{3}\left(1+B^{2} r^{2}\right)^{6}}
$$




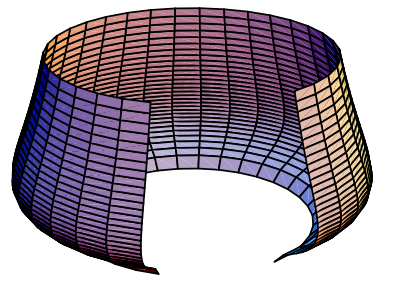

$$
B=0.2
$$
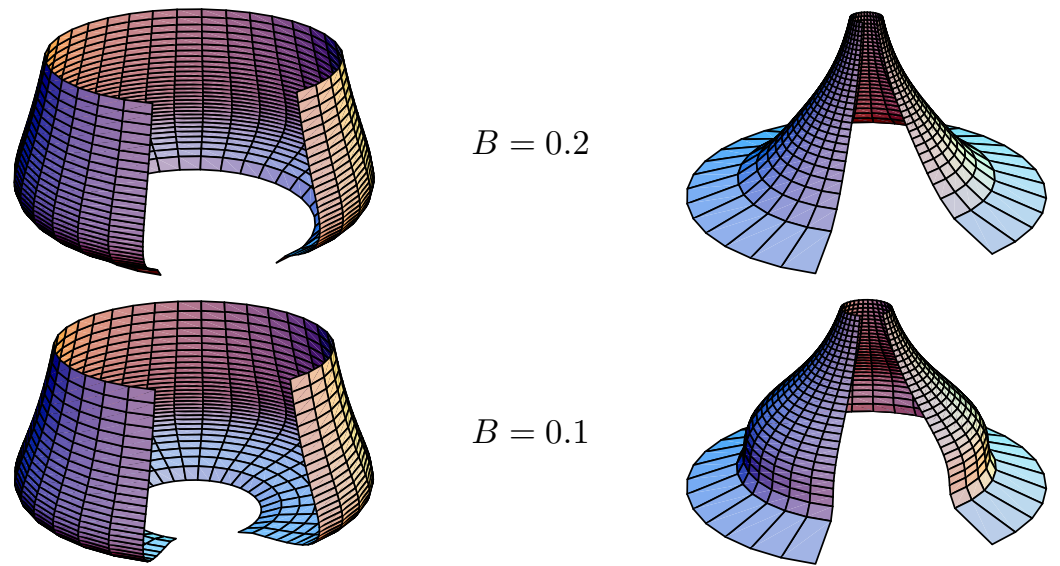

$B=0.1$
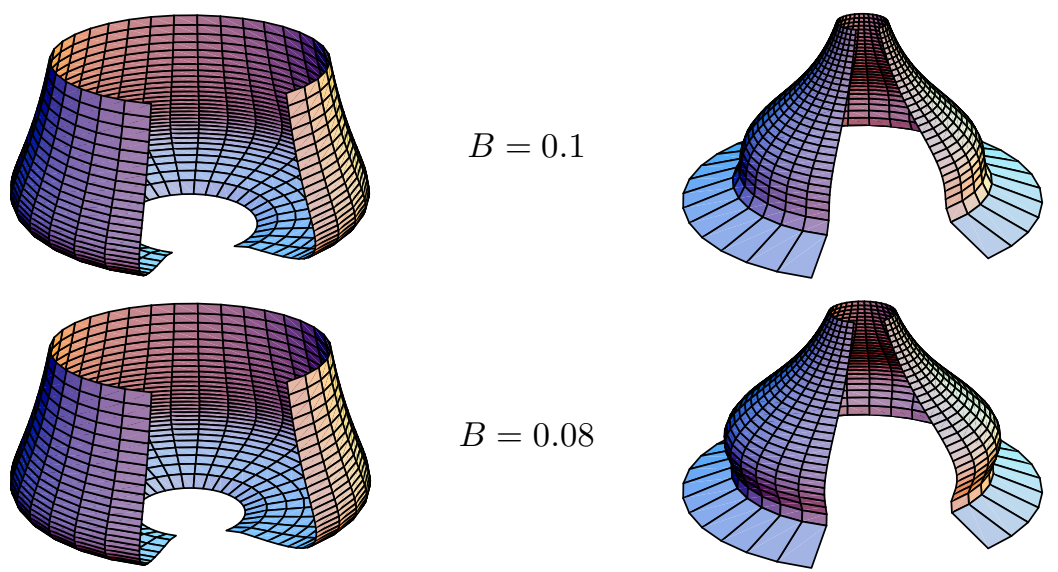

$B=0.08$
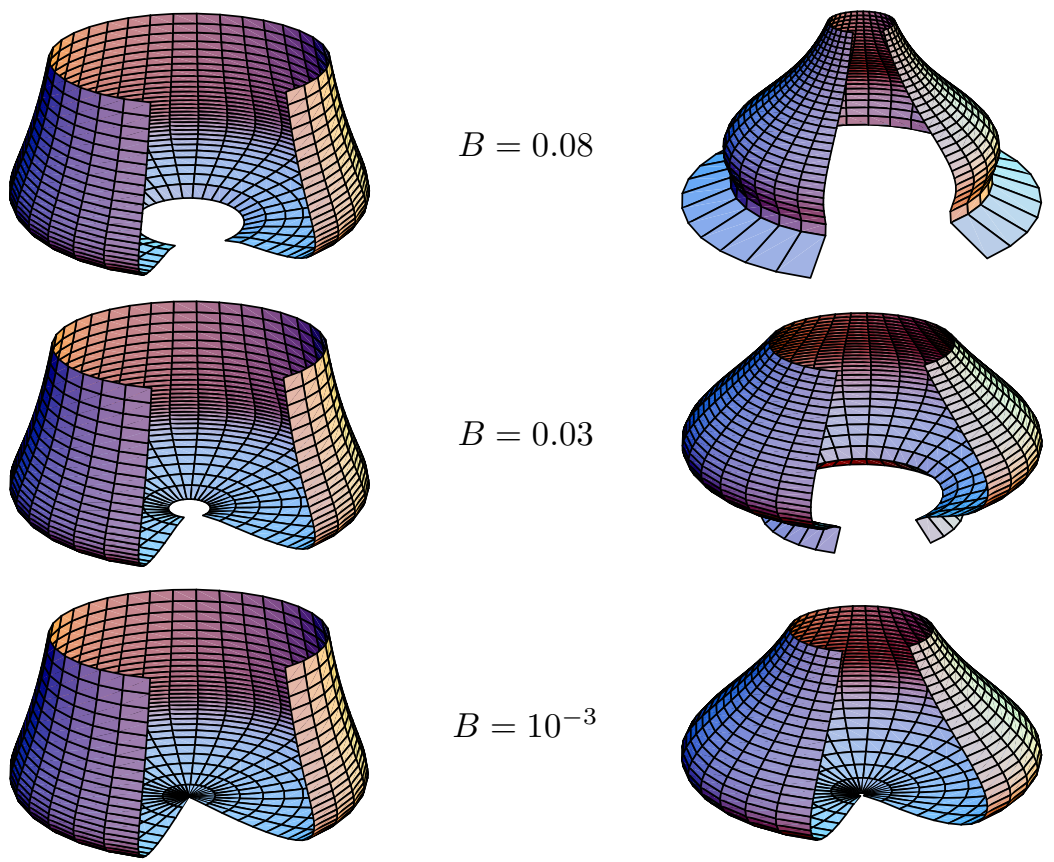

$B=0.03$

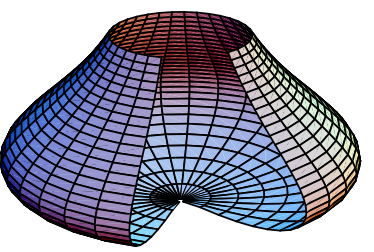

Figure 4. Series of embedding diagrams of the ordinary space (left column) and the optical space (right column). From the optical embeddings one can easily see that for $B>B_{\mathrm{c}}$ (first two top rows) no circular photon orbits exist, for $B<B_{\mathrm{c}}$ (third and fourth row from top) and $B \ll B_{\mathrm{c}}$ (bottom row) stable and unstable circular photon orbit exist. In the last case, the opening and the 'fold' in the lower part of the 'cup', representing the unstable circular orbit are extremally small and beyond the image resolution. Note that both $\rho$ and $z$ scales are adjusted so that all the plots occupy the same area. For real proportions, see Fig. 5 (ordinary embeddings) and Fig. 6 (optical embeddings). 


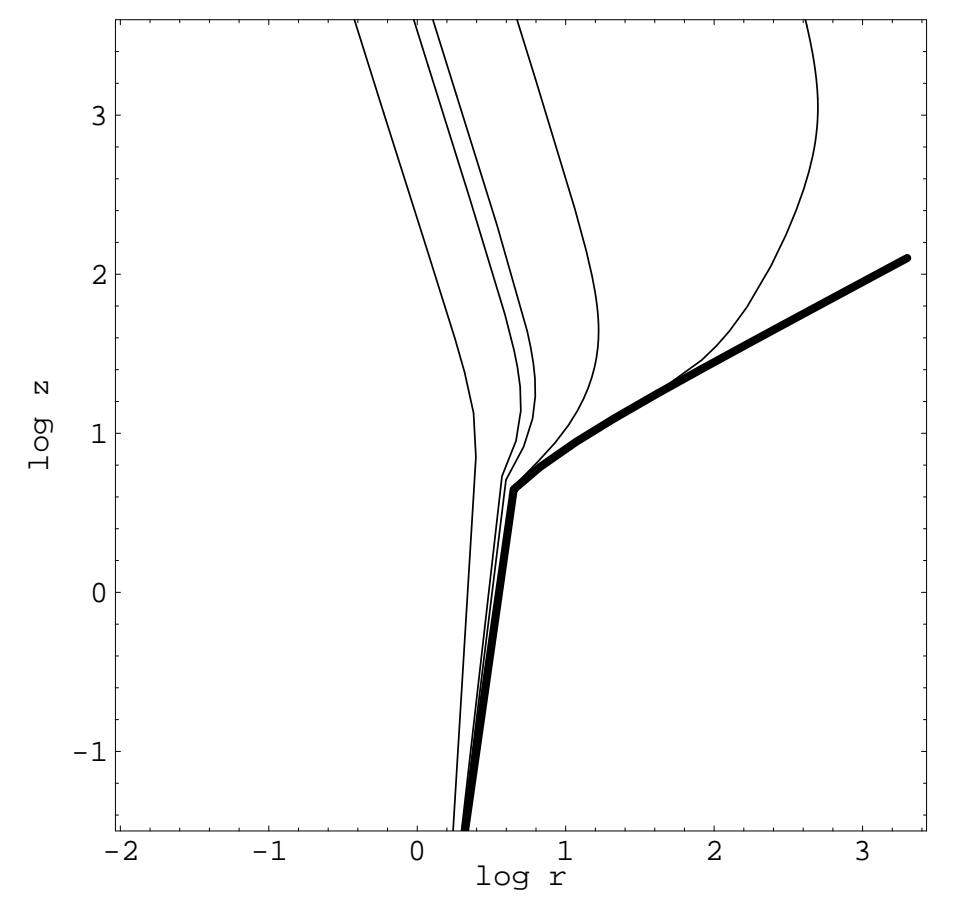

Figure 5. The behaviour of the embedding function $z(\rho)$ for Ernst ordinary space (axial cuts of the diagrams in the left column of Fig. 4 thin curves) in comparison with the pure Schwarzschild case (bold curve), in log-log coordinates that enable to show the order differences in size. The Ernst embedding functions are given (left to right) for the following values of the parameter $B: 0.2,0.1,0.08,0.03$, and $10^{-3}$.

The embedding condition

$$
\left(1-\frac{2}{r}\right)\left(1+B^{2} r^{2}\right)^{6}-\left[1-\frac{3}{r}+B^{2} r(5-3 r)\right]^{2} \geq 0
$$

can be treated numerically only. For $B=0$ it is reduced to the well-known Schwarzschild condition $r>9 / 4$. The numerically obtained results giving the radii of the limit of embeddability $r_{\mathrm{e}}$, are illustrated in Fig. 1. The embedding diagrams are drawn in Fig. 4 for some typical values of the parameter $B$, emphasizing qualitatively different behaviour. For comparison with the Schwarzschild case, we give the functions $z(\rho)$ in Fig. 6. Notice that the turning points of the embedding diagrams correspond just to the circular photon orbits.

In Fig. 7 we give the radii $r_{\mathrm{i}}, r_{\mathrm{o}}, r_{\max }$, and $r_{\mathrm{e}}$ in terms of the Euclidean radial coordinate $\rho$. We can see that the outer turning point of the embedding diagrams, corresponding to $r_{\mathrm{o}}$, is located behind the limit of embeddability, corresponding to $r_{\mathrm{e}}$, if $B<B_{\mathrm{o}} \sim 0.055<B_{\mathrm{c}}$.

\section{Concluding remarks}

We have demonstrated through the analysis of the structure of the photon capture cones and the embedding diagrams of both the ordinary and optical geometry 


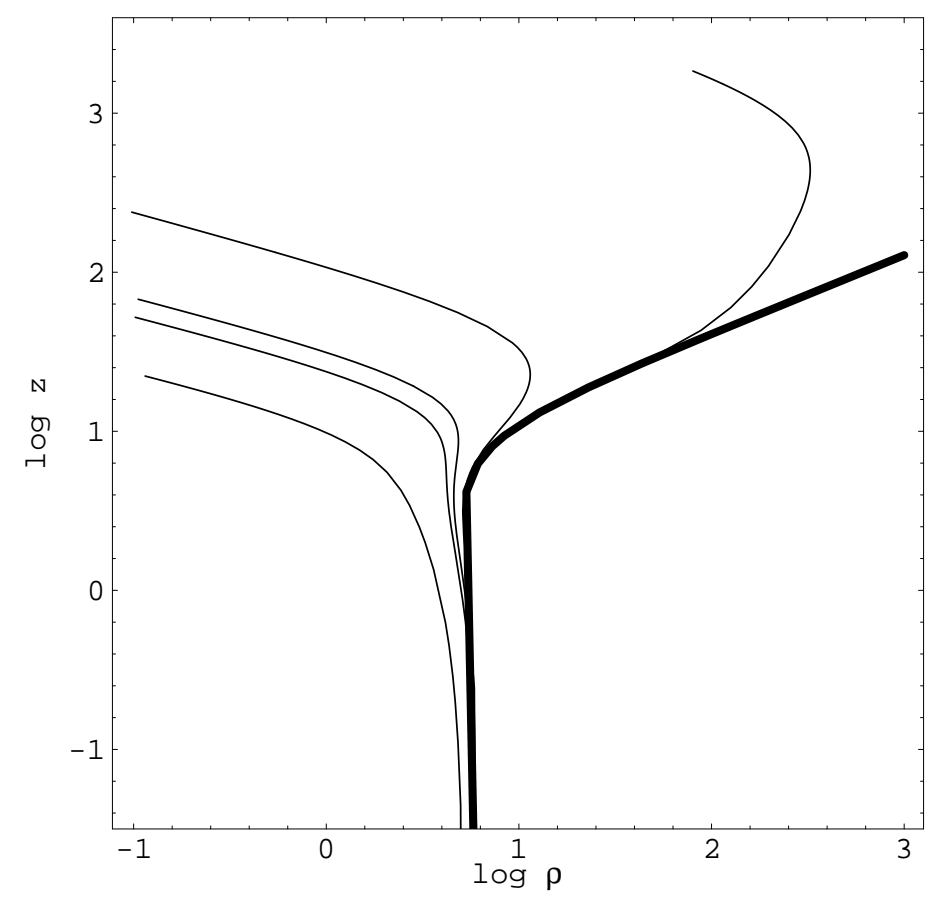

Figure 6. The behaviour of the embedding function $z(\rho)$ for the optical space (axial cuts of the diagrams in the right column of Fig. 14 thin curves) in comparison with the pure Schwarzschild case (bold curve), in log-log coordinates that enable us to show the order differences in size. The Ernst embedding functions are given (left to right) for the following values of the parameter $B: 0.2,0.1,0.08,0.03$, and $10^{-3}$.

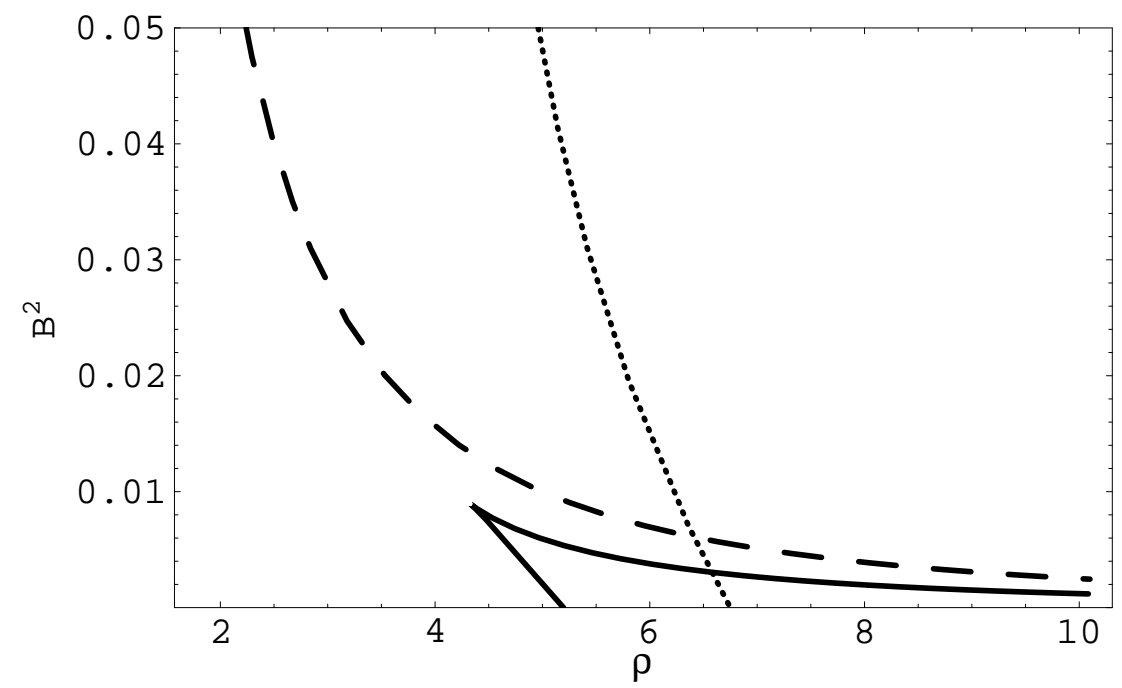

Figure 7. Same as in Fig. 1 in terms of the Euclidean coordinate $\rho$. 
that the Ernst spacetimes have some highly different properties in comparison with Schwarzschild spacetime.

The global properties of the Ernst spacetimes are imprinted in the character of the photon capture cones of static observers at each radius $r>2$. Namely, the asymptotic structure of the Ernst spacetimes reflects itself in the fact that at any radius above the event horizon only the purely radial outward directed photons can escape to infinity. This fact, together with the existence of captured photons at $r_{\mathrm{i}}<r<r_{\mathrm{O}}$, makes the structure of the photon capture cones in the Ernst spacetimes to be completely different from the limiting case of the Schwarzschild spacetime at any radius above the event horizon.

Moreover, the character of the embedding diagrams of both the ordinary and optical geometry (Figs [5] and [6) reflecting locally the gravitational and inertial phenomena (see, e.g., 7]), indicates that these phenomena have to be qualitatively different from the Schwarzschild ones at all $r>2$ for $B \sim 1$, and at $r>1 / B$ for $B \ll 1$.

\section{Acknowledgments}

The present work was supported by the GAČR grant No. 202/96/0206 and by the Abdus Salam International Centre for Theoretical Physics in Trieste. Its important part has been done during the visit of the authors at the ICTP. The authors would like to thank Prof. M. A. Abramowicz, Prof. D. Sciama and the Head of the High Energy Sector, Prof. S. Randjbar-Daemi for kind hospitality at the ICTP.

\section{References}

[1] Ernst F J 1976 J. Math. Phys. 1754

[2] Dadhich N, Hoenselars C and Vishweshwara C V 1979 J. Phys. A 12, 215

[3] Esteban E P 1984 Nuovo Cimento B $\mathbf{7 9} 76$

[4] Esteban E P 1985 Nuovo Cimento B 8735

[5] Aliev A N and Galtsov D V 1989 Sov. Phys. Usp. 3275

[6] Karas V and Vokrouhlický D 1990 Class. Quantum Grav. 7391

[7] Misner C W, Thorne K S and Wheeler J A 1973 Gravitation (San Francisco: W. H. Freeman and Company)

[8] Carter B 1973 in Black Holes ed C De Witt and B S De Witt (New York: Gordon and Breach)

[9] Kristiansson S, Sonego S and Abramowicz M A 1998 Gen. Rel. Grav. 30275

[10] Stuchlík Z and Hledík S 1998 Some properties of the Schwarzschild-de Sitter and Schwarzschildanti-de Sitter spacetimes, preprint TPA 010 (1999), Silesian University at Opava

[11] Abramowicz M A, Carter B and Lasota J P 1988 Gen. Rel. Grav. 201173

[12] Abramowicz M A and Prasanna A 1990 Mon. Not. R. Astron. Soc. 245720

[13] Abramowicz M A 1990 Mon. Not. R. Astron. Soc. 245733

[14] Abramowicz M A 1992 Mon. Not. R. Astron. Soc. 256710

[15] Stuchlík Z 1990 Bull. Astron. Inst. Czechoslov. 41341

[16] Abramowicz M A, Miller J C and Stuchlík Z 1993 Phys. Rev. D 471440 